\title{
Soluble Tumor Necrosis Factor (TNF) Receptors (sTNF-R1 and -R2) in Pregnant Women Chronically Infected with Trypanosoma cruzi and their Children
}

\author{
Miriam Martin García, Ana Maria De Rissio, Ximena Villalonga, Eleonora Mengoni, and Rita L. Cardoni* \\ Instituto Nacional de Parasitología "Dr. M. Fatala Chabén,” Administración Nacional de Laboratorios e Institutos de Salud \\ "Dr. CG Malbrán” (ANLIS), Buenos Aires, Argentina
}

\begin{abstract}
Most congenital transmissions of Trypanosoma cruzi are not detected. As the levels of mediators regulating the immune response might be different in the absence or in the presence of transmission, we explored the levels of tumor necrosis factor (TNF) and soluble TNF receptors TNF-R1 and -R2 in T. cruzi-infected pregnant women and the neonates. We previously found that the circulating levels of TNF were higher in non-transmitting than in transmitting pregnant women. This observation has now been extended to the spontaneous release of TNF by peripheral blood leukocytes (PBLs) that was also higher in non-transmitting than in transmitting pregnant women. As their mothers, non-infected neonates had higher circulating levels of TNF than congenitally infected children. The circulating levels of sTNF-R1 increased in non-transmitting and transmitting mothers and in infected and non-infected neonates. The circulating levels of sTNF-R2 were $\sim 60 \%$ higher in infected than in non-infected neonates $(1,635 \pm 101$ and $1,027 \pm 100$ $\mathrm{pg} / \mathrm{mL}$, respectively) and remained higher at 1 year of age. This important increase, only observed in infected neonates, could be useful to orientate to the presence of vertical transmission of $T$. cruzi infection.
\end{abstract}

\section{INTRODUCTION}

In Argentina, with $\sim 736,000$ births per year (population $=$ 37,870,000), 182 cases of congenital Trypanosoma cruzi infection, the parasite that causes Chagas disease, are annually detected. However, it is estimated that for each of them, 6-12 infected neonates are not diagnosed. ${ }^{1,2}$ In the non-endemic areas for T. cruzi infection, such as Buenos Aires and surrounding cities, $6-12 \%$ of women that deliver their children in public hospitals are infected with $T$. cruzi. Vertical transmission is detected in $1-4 \%$ of these cases, and these infections cannot be prevented with trypanocidal treatment because of the theratogenic effects of the available drugs. ${ }^{3-6}$

The early diagnosis of $T$. cruzi infection in the neonates is of great importance to optimize their treatment. ${ }^{7}$ Thus, it is important to improve the diagnosis of the congenitally acquired infection. Transmission is at least related with an increased maternal parasite burden, although it is very low in both transmitting and non-transmitting chronically infected women. ${ }^{8}$ We explored the relationship among the levels of mediators regulating the inflammatory response and the congenital transmission of $T$. cruzi infection. The production of tumor necrosis factor (TNF) and some of the TNF regulators, such as soluble TNF receptors (sTNF-R1 and -R2), as well as interleukin- (IL-)10, was studied in T. cruzi-infected pregnant women and their children.

In $T$. cruzi infections TNF might have a dual role. In the acute phase of murine infection, TNF has mainly a protective activity because it is an important stimulator, besides $\gamma$ interferon, of the NO-mediated trypanocidal mechanism. ${ }^{9,10}$ However, Chagas disease is characterized by inflammatory reaction affecting host tissues, not only in heart and skeletal muscle ${ }^{11}$ but also in placenta, ${ }^{6}$ and TNF might also be engaged in this host response. ${ }^{12}$ TNF exerts its activity on interaction with two trans-membrane glycoprotein receptors: TNF-R1 (p55) and -R2 (p75). The soluble forms of both receptors, s-TNF-R1 and -R2, bind TNF with high affinity, transducing or inhibiting TNF biologic activity. At high con-

* Address correspondence to Rita L. Cardoni, Av Paseo Colon 568, Buenos Aires 1426, Argentina. E-mail: rlcardoni@yahoo.com centrations, they inhibit TNF bioactivity by competing with the membrane receptors. At low concentrations, they could stabilize the trimeric TNF structure increasing its half-life and acting as a reservoir of TNF. ${ }^{13}$ IL-10 is also an important regulator of TNF production and the inflammatory response. We previously found that circulating levels of IL-10 did not increase in pregnant women chronically infected with $T$. cruzi comparing them to women only pregnant or only infected. In addition, a decrease of the circulating levels of TNF associated to normal pregnancy also occurred in transmitting but not in non-transmitting $T$. cruzi-infected women. ${ }^{14}$ Here we compared the spontaneous production of TNF, sTNF-R1, and -R2 and IL-10 by peripheral blood leukocytes (PBLs) as well as the circulating levels of mediators in transmitting and nontransmitting mothers. The increase in the levels of circulating $\mathrm{TNF}^{14}$ was associated with the increase of the spontaneous release of TNF by PBLs of non-transmitting compared with transmitting pregnant women. Mirroring the differences in their mothers, circulating levels of TNF were higher in noninfected than in $T$. cruzi congenitally infected neonates. The levels of sTNF-R1 increased in mothers and neonates, whether they were infected or not. On the other hand, an important increase in the level of sTNF-R2 was observed only in infected children and, in the non-treated cases, sTNF-R2 level remained high at 1 year of age. Thus, differences in TNF and their regulators could help to find elements associated to vertical transmission of $T$. cruzi infection.

\section{MATERIALS AND METHODS}

Subjects. The diagnosis of $T$. cruzi infection in pregnant women and children was carried out in the Instituto Nacional de Parasitología "Dr. M. Fatala Chabén," the reference center for the diagnosis in Argentina. Groups of pregnant women infected or not with $T$. cruzi, as well as infected and noninfected women without clinically recognized pregnancy, were selected at random. All the infected women and children included were residents in the non-endemic area and did not receive a blood transfusion in the last year before the study. They received medical assistance in public hospitals located in non-endemic urban areas of Buenos Aires and surrounding cities. According to the clinical evaluation, which 
included an electrocardiogram and thorax x-rays, women were in the asymptomatic phase of the chronic $T$. cruzi infection, and none of them had been treated with trypanocidal drugs. Venous blood was withdrawn with and without anticoagulant. Sera were used for serologic assays. Plasma was kept at $-70^{\circ} \mathrm{C}$. The Ethical Committee of the ANLIS approved this study, and informed written consent was obtained for all participants.

Diagnosis of $\boldsymbol{T}$. cruzi infection. The presence of specific antibodies in sera was determined by indirect hemagglutination (IHA), indirect immunofluorescence (IFA), and enzymelinked immunosorbent assays (ELISA). ${ }^{15}$ For IHA, the samples were incubated with fixed red cells coated with $T$. cruzi antigens. For IFA, the samples and a fluorescein isothiocyanate (FITC)-labeled antibody anti-human IgG (Sigma, St. Louis, MO) were incubated on smears of T. cruzi epimastigotes. IHA and IFA were carried out with 2 -fold serial dilutions of sera. The ELISA was carried out with a 1/200 dilution of the samples incubated in microplates precoated with $T$. cruzi epimastigotes antigens. The binding of specific antibodies was detected with a horseradish peroxidase (HRP)labeled antibody anti-human IgG (Sigma). After the addition of the substrate $o$-phenylenediamine (OPD; Sigma), the optical density at $490 \mathrm{~nm}$ (OD $490 \mathrm{~nm}$ ) was quantified in an ELISA Reader (Model 550; BIO RAD, Tokyo, Japan). Women were considered infected when at least two of the serologic tests were reactive, according to the criteria of the World Health Organization and the Argentinean guidelines. ${ }^{16}$

The presence of $T$. cruzi in the blood of children was determined in $0.5-1.5 \mathrm{~mL}$ of heparinized blood that was centrifuged in Eppendorf tubes. The buffy coat was distributed in six slides and examined carefully by light microscopy at $\times 400$ for at least 30 minutes. ${ }^{16}$ The assay was carried out in the first month after birth and, when the parasite was not detected, the test was repeated at 6 and 12 months of age. The serologic assays described above were also carried out to evaluate the presence of specific antibodies. The children with positive controls were referred for treatment of $T$. cruzi infection.

Blood culture. Heparinized blood was diluted 1/10 in RPMI medium (Sigma) containing HEPES buffer, $50 \mu \mathrm{mol} / \mathrm{L} 2$-mercaptoethanol, $100 \mathrm{IU}$ penicillin, and $100 \mu \mathrm{g} / \mathrm{mL}$ streptomycin. After 24 hours of culture in a $\mathrm{CO}_{2}$ incubator, cells were centrifuged at $400 \mathrm{~g}$ for 5 minutes, and the cell-free supernatants were fractionated and kept at $-70^{\circ} \mathrm{C} .^{17}$

Levels of TNF, sTNF-R1, sTNF-R2, and IL-10. Levels were determined by two-site ELISA assays by using commercially available kits for TNF, sTNF-R1 and IL-10 (OptEIA Kit; BD PharMingen, San Jose, CA), as well as for sTNF-R2 (R\&D Systems, Minneapolis MN), according to the manufacturer's protocols. Briefly, an antibody specific for the corresponding cytokine was adsorbed to high-binding microplates (Costar Corp., Cambridge, MA). The plates were washed with $0.05 \%$ Tween 20-phosphate-buffered saline (PBS) and blocked with $1 \%$ bovine serum albumin-PBS. After the addition of $100 \mu \mathrm{L}$ of samples, the plates were incubated overnight. They were washed and incubated with a biotinylated detection antibody and avidin-HRP conjugate. The OD $490 \mathrm{~nm}$ obtained after the addition of OPD was measured. The OD $490 \mathrm{~nm}$ mean values of duplicates of the samples were interpolated in the standard curves. The Log transformation of the standard concentration versus OD $490 \mathrm{~nm}$ was lineal up to $500 \mathrm{pg} / \mathrm{mL}$. The sensitivity of the assays was $4 \mathrm{pg} / \mathrm{mL}$ for IL-10 and $2 \mathrm{pg} / \mathrm{mL}$ for TNF, sTNF-R1, and sTNF-R2. Internal standards indicated that the presence of either sTNF-R1 or sTNF-R2 did not interfere with TNF determinations. In addition, the presence of TNF did not interfere with either sTNF-R1 or sTNFR2 determinations, as previously reported. ${ }^{18}$

Statistical analysis. Differences among groups were examined either by Mann-Whitney test or by Kruskal-Wallis test and Dunn post-test for multiple comparisons, using XLStat software. Discontinuous variables were normalized by logarithmic transformation. $P<0.05$ was considered significant.

\section{RESULTS}

The groups of non-infected and T. cruzi-infected women as described in Table 1 were studied. The infected children were diagnosed in the first (nine cases) or second (five cases) parasitologic control and those with undetectable parasitemia by serologic test after 10 months of age (six cases). Non-infected newborns born to $T$. cruzi-infected mothers had three negative parasitologic controls, as well as negative serologic tests carried out after 10 months of age. None of the children studied were premature. Their weights at birth were $3.3 \pm 0.5$ (range, $1.9-4.1 \mathrm{~kg}$ ) and $3.4 \pm 0.4 \mathrm{~kg}$ (range, $2.6-4.3 \mathrm{~kg}$ ) for infected and non-infected babies, respectively $(P>0.05$, Mann-Whitney test).

In the groups of women studied, the plasma levels of TNF were higher in non-transmitting than in transmitting T. cruziinfected women, as previously described. ${ }^{14}$ Non-transmitting mothers also had higher levels of sTNF-R1 than the transmitting group. However, in both groups of infected pregnant women, the levels of sTNF-R1 were higher than the values in non-pregnant non-infected women (Table 2).

TABLE 1

Characteristics of the groups of women studied

\begin{tabular}{|c|c|c|c|c|c|}
\hline & \multicolumn{2}{|c|}{ Non-infected } & \multicolumn{3}{|c|}{ Infected with $T$. cruzi } \\
\hline & $\begin{array}{c}\text { Non-pregnant } \\
(N=24)\end{array}$ & $\begin{array}{l}\text { Pregnant } \\
(N=24)\end{array}$ & $\begin{array}{l}\text { Non-pregnant } \\
\quad(N=24)\end{array}$ & $\begin{array}{l}\text { Pregnant non-transmitting } \\
\qquad(N=24)\end{array}$ & $\begin{array}{c}\text { Pregnant transmitting } \\
(N=20)\end{array}$ \\
\hline Age (years) & $27 \pm 8$ & $26 \pm 6$ & $30 \pm 6$ & $30 \pm 7$ & $26 \pm 6$ \\
\hline Age range (years) & $(17-44)$ & $(15-39)$ & $(21-42)$ & $(20-42)$ & $(16-37)$ \\
\hline Time of pregnancy (months) & - & $5.7 \pm 2.0$ & - & $6.7 \pm 1.4$ & $5.6 \pm 1.4$ \\
\hline \multicolumn{6}{|c|}{ Serologic tests of T. cruzi infection: } \\
\hline IHA & $3.2 \pm 0.7$ & $3.4 \pm 0.8$ & $7.6 \pm 0.8$ & $6.8 \pm 1.2$ & $7.5 \pm 1.1$ \\
\hline IFA & $3.1 \pm 0.6$ & $3.0 \pm 0.0$ & $6.8 \pm 0.6$ & $6.8 \pm 0.5$ & $6.9 \pm 0.5$ \\
\hline ELISA & $44 \pm 29$ & $48 \pm 28$ & $359 \pm 96$ & $326 \pm 71$ & $353 \pm 87$ \\
\hline
\end{tabular}

The diagnosis of T. cruzi infection was carried out as detailed in Materials and Methods. The results of indirect immunohemagglutination (IHA) and indirect immunofluorescence assays (IFA) are expressed as the $\log _{2}$ of the inverse of the titer and considered reactive when $\geq 5$. ELISA is expressed as the optical density at $490 \mathrm{~nm} \times 1,000$ and considered reactive when $\geq 200$. The samples were obtained at the specified time of pregnancy. No significant differences were detected in the results of the serologic tests among the infected groups (Kruskal-Wallis test and Dunn post-test). Mean values \pm SD are shown. 
TABLE 2

Levels of circulating sTNF-R1 and sTNF-R2 in pregnant women chronically infected with T. cruzi

\begin{tabular}{|c|c|c|c|c|}
\hline & \multirow{2}{*}{\multicolumn{2}{|c|}{ Non-infected }} & \multicolumn{2}{|c|}{ Infected } \\
\hline & & & \multirow[b]{2}{*}{ Non-pregnant } & \multirow{2}{*}{$\begin{array}{l}\text { Pregnant (transmitting } \\
\text { and non-transmitting) }\end{array}$} \\
\hline & Non-pregnant & Pregnant & & \\
\hline sTNF-R1 (pg/mL) & $761 \pm 68(14)$ & $970 \pm 76(14)$ & $821 \pm 52(13)$ & $1053 \pm 60 *(27)$ \\
\hline sTNF-R2 (pg/mL) & $1174 \pm 72(16)$ & $1168 \pm 50(16)$ & $1164 \pm 65(16)$ & $1351 \pm 52(31)$ \\
\hline Blood leukocytes $\left(\mathrm{N} / \mathrm{mm}^{3}\right)$ & $6410 \pm 280(17)$ & $7600 \pm 310(19)$ & $6020 \pm 430(21)$ & $7780 \pm 540 *(17)$ \\
\hline Monocytes (\%) & $7.2 \pm 0.5(16)$ & $7.5 \pm 0.5(14)$ & $6.7 \pm 0.7(16)$ & $8.2 \pm 0.6(15)$ \\
\hline
\end{tabular}

${ }^{*}$ The infected pregnant women had significantly higher levels of sTNF-R1 and circulating leukocytes than non-infected non-pregnant women, $P<0.05$, Kruskal-Wallis test and Dunn post-test. In infected pregnant women, the number of blood leukocytes and the percentage of monocytes were similar between $T$. cruzi transmitting and non-transmitting mothers, Mann-Whitney test. Mean values \pm SEM are shown. In parentheses: number of cases.

On the other hand, the circulating levels of sTNF-R2 were not affected by pregnancy or T. cruzi infection (Table 2). In addition, non-significant differences were found in the circulating levels of sTNF-R2 (Figure 1) and IL-10 ${ }^{14}$ between transmitting and non-transmitting mothers.

The release of the same mediators by in vitro cultured PBLs is shown in Figure 1B. In T. cruzi-infected pregnant women, the spontaneous release of TNF was higher in nontransmitting than in transmitting mothers, as occurred with the circulating levels. On the other hand, we could not detect significant differences in the levels of sTNF-R1, sTNF-R2,

\section{A) Circulating}

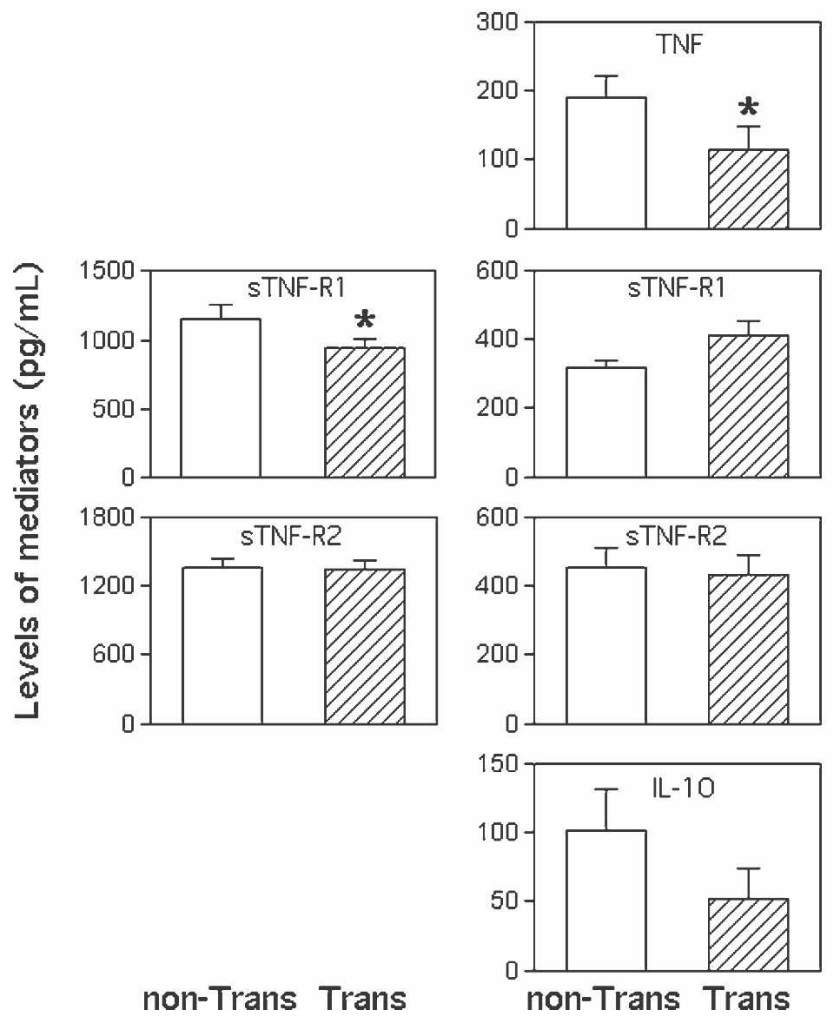

FigurE 1. Circulating levels and spontaneous release of mediators in pregnant women chronically infected with T. cruzi. Plasma levels (A) and/or spontaneous release (B) of TNF, sTNF-R1, sTNF$\mathrm{R} 2$, and IL-10 by in vitro cultured circulating leukocytes. Mean values \pm SEM is shown. *The release of TNF was lower in transmitting (Trans, striped bars) than in non-transmitting women (non-Trans, open bars), $P<0.05$, Mann-Whitney test. In addition, the circulating level of sTNF-R1 was higher in non-transmitting than in transmitting women, $P<0.05$, Mann-Whitney test. and IL-10 released by PBLs between the two groups. The number of PBLs increased in infected pregnant women (Table 2). However, the number of PBLs and the percentage of monocytes that could produce the mediators evaluated ${ }^{18}$ were similar in the transmitting and non-transmitting groups of women (monocytes: $7.6 \pm 1.6 \%$ and $8.4 \pm 0.8 \%$, respectively; $P>0.05$, Mann-Whitney test).

The levels of mediators were also evaluated in children born to $T$. cruzi-infected mothers. In the case of infected children, studies were performed before the administration of the trypanocidal treatment. In the first months of age, infected babies had, as transmitting mothers, lower levels of TNF than the non-infected babies (Figure 2). The circulating levels of sTNF-R1 were similar in infected and non-infected babies. In the first month, however, sTNF-R1 was higher than at 6 and 12 months of age in both groups of neonates $(P<$ 0.05 , Kruskal-Wallis test). The levels of sTNF-R2 were higher in infected than in the non-infected babies and remained higher at 1 year of age. Circulating IL-10 had similar levels in both groups of neonates.

\section{DISCUSSION}

Normal pregnancy is characterized by a downregulation of both the Th1 response and the release of inflammatory mediators. ${ }^{19,20}$ Although the inflammatory response is required for the control of infections, experimental and clinical evidence has shown that the downregulation of the inflammatory mediators is an important component of normal pregnancy. In the absence of infections, an increased production of TNF and other inflammatory cytokines by fetal membranes seems to be the signal for term labor and delivery. ${ }^{21}$ In response to intrauterine infections, maternal tissues, such as decidua, can produce inflammatory mediators. To avoid premature labor, the elevated production of inflammatory cytokines has to be controlled with an activation of mechanisms regulating the biologic activities of TNF. ${ }^{22}$ Steroids, which are present in circulation at high levels throughout pregnancy, are potential candidates for regulating TNF potential. The TNF secretion by stimulated monocyte/macrophages is inhibited in vivo and in vitro by glucocorticoids. ${ }^{23}$ In addition, pregnancy-specific glycoproteins, derived from placenta, induce the secretion of anti-inflammatory cytokines, such as IL-10. ${ }^{24}$ In vitro, IL-10 downregulates TNF activity in three ways: by inhibiting the release of TNF, by increasing the release of sTNF-R1 and -R2, and by concomitantly reducing surface expression of both TNF receptors. ${ }^{19}$ The increase of soluble TNF receptors is also induced by progesterone, because the deficiency in the circulating s-TNF-R1 and -R2 of women with recurrent spon- 

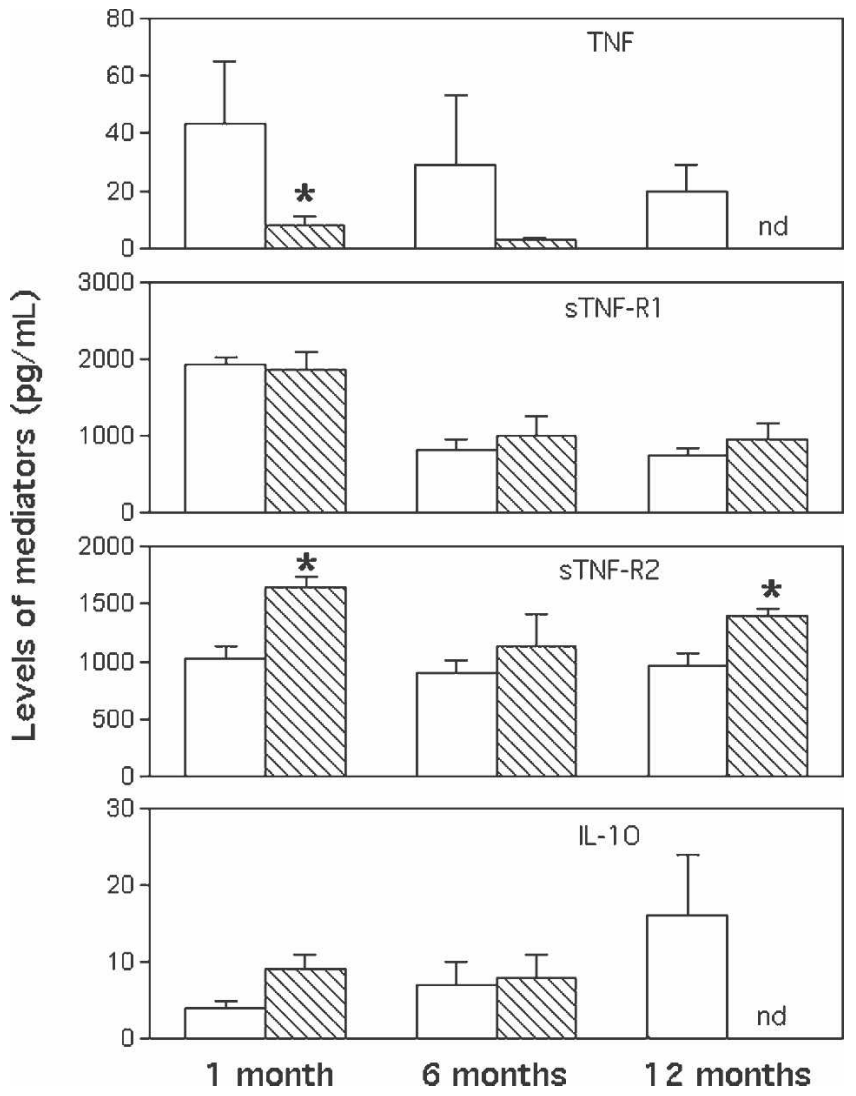

FIGURE 2. Circulating levels of mediators in children born to $T$. cruzi-infected mothers. Levels of TNF, sTNF-R1, sTNF-R2, and IL10 in $T$. cruzi-infected (striped bars) and non-infected (open bars) children at 1,6 , and 12 months of age. Mean values \pm SEM is shown. *The differences in the levels of TNF (at 1 month of age) as well as in sTNF-R2 (at 1 and 12 months of age) between infected and noninfected children are significant, $P<0.05$, Mann-Whitney test. In both, infected and non-infected neonates, the level of sTNF-R1 in the first month was higher than at 6 and 12 months of age $(P<0.05$, Kruskal-Wallis test). nd, not done.

taneous abortion can be restored by progesterone treatment. $^{25}$

We previously found a downregulation of the circulating levels of TNF in transmitting, but not in non-transmitting $T$. cruzi-infected pregnant women. ${ }^{14}$ The in vitro spontaneous release of TNF by PBLs was, in agreement with the circulating levels, lower in T. cruzi-transmitting than in nontransmitting women, suggesting the participation of PBLs in the release of TNF. In addition, non-infected neonates, born to $T$. cruzi-infected mothers, had higher levels of TNF than infected children.

To our knowledge, production of TNF by transmitting and non-transmitting $T$. cruzi-infected mothers and their neonates has not been compared by other groups. However, it was previously observed that the TNF production by PBLs of $T$. cruzi-infected non-transmitting mothers and their children were higher than in non-infected mothers and their children, with in vitro specific and non-specific stimulation. ${ }^{26}$

The increase in the levels of TNF we found in nontransmitting infected mothers was very mild compared with the amounts observed in other parasitic infections, such as malaria and visceral Leishmaniasis, ${ }^{27,28}$ but could be ascribed to the chronicity of $T$. cruzi infection in this asymptomatic population. However, this increase observed in nontransmitting mothers and their neonates could contribute to the control of the infection to avoid vertical transmission.

TNF is mainly produced by activated macrophages. However, endothelial cells, fibroblasts, and $\mathrm{B}$ and $\mathrm{T}$ lymphocytes are also a source of TNF. Considering the tissular localization of the main TNF producers, a focused TNF release may not be reflected in circulation. Such compartmentalized TNF increase could explain the high amounts of soluble TNF receptors we found in $T$. cruzi-infected mothers and children. In this regard, TNF is increased in amniotic fluid, but not in serum, in pregnant patients with infections and premature rupture of membranes. ${ }^{29}$

TNF is required for the control of infections, but at high amounts, TNF could be deleterious for the host. The role of soluble TNF receptors as a defense mechanism against the excess of TNF has been observed in the experimental models of sepsis. ${ }^{30}$ In uncomplicated pregnancy, the increase of soluble TNF receptors, detected in serum and amniotic fluid, as well as in urine, seems to protect the fetus against TNF. ${ }^{31}$ We found a moderate increase in sTNF-R1 levels in transmitting and non-transmitting T. cruzi-infected mothers and their neonates, whether they were infected or not, that could provide the regulation required for TNF activity.

Strikingly, the increase in circulating sTNF-R2 observed in the infected neonates was of similar magnitude to that observed in sepsis and in the experimental administration of lipopolysaccharide (LPS) to normal subjects. In experimental sepsis, TNF can reach $400 \mathrm{pg} / \mathrm{mL}$, and sTNF-R2 increases from basal levels of $\sim 800-1,000$ to $1,400 \mathrm{pg} / \mathrm{mL}$ in plasma. $^{30}$ The levels of sTNF-R1 and sTNF-R2 also increase $~ 50 \%$ in neonates with sepsis/pneumonia. ${ }^{32}$

Alterations in the control of the inflammatory response could have consequences to the congenital transmission and to the children's response, even when the role of TNF and the regulation of TNF levels in vertical transmission are unclear. The sTNF-R2 increase in the infected neonates could protect the children from the inflammatory response induced by $T$. cruzi, but disregarding the functional role for the host, the high levels of sTNF-R2 could orientate to the presence of mother to child transmission of T. cruzi infection. Taking into account that most of the cases of congenitally transmitted $T$. cruzi infection are not diagnosed, ${ }^{1,2}$ and the striking high congenital estimated prevalence for Argentina (1 infection per 400 births), these findings could be very useful for the development of markers of vertical transmission.

Received August 16, 2007. Accepted for publication November 28, 2007.

Acknowledgments: The authors thank M. I. Antúnez and D. L. Boveris for helpful discussion.

Financial support: This work was supported by the Consejo Nacional de Investigaciones Científicas y Técnicas (CONICET), PIA N ${ }^{\circ}$ 5170, Argentina.

Authors' addresses: Miriam Martin García, Ana Maria De Rissio, Ximena Villalonga, Eleonora Mengoni, and Rita L. Cardoni, Instituto Nacional de Parasitología "Dr. M. Fatala Chabén," Av. Paseo Colón 568, 1063 Buenos Aires, Argentina, Tel: 54-11-4331-7732, Fax: 54-11-4331-7142, E-mail: rlcardoni@yahoo.com.

Reprint requests: Rita L. Cardoni, Instituto Nacional de Parasitología "Dr. M. Fatala Chabén," Av. Paseo Colón 568, 1063 Buenos Aires, Argentina, Tel: 54-11-4331-7732, Fax: 54-11-4331-7142, E-mail: rlcardoni@yahoo.com. 


\section{REFERENCES}

1. Schmuñis GA, 1999. A Tripanossomiase Americana e seu impacto na saúde pública das Americas. Brener Z, Andrade ZA, Barral Neto M, eds. Trypanosoma cruzi e Doenca de Chagas. Rio de Janeiro: Guanabara Koogan, 1-15.

2. Gürtler RE, Segura EL, Cohen JE, 2003. Congenital transmission of Trypanosoma cruzi infection in Argentina. Emerg Infect Dis 9: 29-32.

3. Arcavi M, Orfus G, Griemberg G, 1993. Incidencia de la infección chagásica en embarazadas y recién nacidos en área no endémica. Medicina (B Aires) 53: 217-222.

4. Storni P, Bolsi FL, 1979. Embarazo y parasitismo por Trypanosoma cruzi. Medicina (B Aires) 39: 193-197.

5. Szarfman A, Urman J, Otalova A, Larzuia A, Yanovsky JF, 1975. Specific agglutinins and immunoglobulin levels in congenital Chagas disease. Medicina (B Aires) 35: 245-250.

6. Votta RA, Marchese CA, Pastorini A, Gonzalez CA, Lautrec L, Tomassini TL, 1977. Multimetodología diagnóstica de la enfermedad de Chagas Mazza congénita en un medio no endémico. Revista de la Sociedad de Obstetricia y Ginecología de Buenos Aires 56: 237-250.

7. Freilij H, Altcheh J, 1995. Congenital Chagas disease: diagnosis and clinical aspects. Clin Infect Dis 21: 551-555.

8. Hermann E, Truyens C, Alonso Vega C, Rodriguez P, Berthe A, Torrico F, Carlier Y, 2004. Congenital transmission of Trypanosoma cruzi is associated with maternal enhanced parasitemia and decreased production of Interferon- $\gamma$ in response to parasite antigens. J Infect Dis 189: 1274-1281.

9. Santos Lima EC, García I, Vicentelli MH, Vassalli P, Minoprio P, 1997. Evidence for a protective role of tumor necrosis factor in the acute phase of Trypanosoma cruzi infection in mice. Infect Immun 65: 457-465.

10. Hölscher C, Köhler G, Müller U, Mossmann H, Schaub GA, Brombacher F, 1998. Defective nitric oxide effector functions lead to extreme susceptibility of Trypanosoma cruzi-infected mice deficient in gamma interferon receptor or inducible nitric oxide synthase. Infect Immun 66: 1208-1215.

11. Köberle F, 1968. Chagas' disease and Chagas' syndromes: the pathology of American trypanosomiasis. Adv Parasitol 6: 63116.

12. Perez-Fuentes R, Guegan JF, Barnabe C, Lopez-Colombo A, Salgado-Rosas H, Torres-Rasgado E, Briones B, Romero-Díaz M, Ramos-Jimenez J, Sánchez-Guillen M del C, 2003. Severity of chronic Chagas disease is associated with cytokine/ antioxidant imbalance in chronically infected individuals. Int $J$ Parasitol 33: 293-299.

13. Derouich-Guergour D, Brenier-Pinchart MP, Ambroise-Thomas P, Pelloux H, 2001. Tumour necrosis factor $\alpha$ receptors: role in the physiopathology of protozoan parasite infections. Int $J$ Parasitol 31: 763-769.

14. Cardoni RL, García MM, De Rissio AM, 2004. Proinflammatory and anti-inflammatory cytokines in pregnant women chronically infected with Trypanosoma cruzi. Acta Trop 90: 65-72.

15. Cura EN, Segura EL, 1998. Quality assurance of the serologic diagnosis of Chagas disease. Rev Panam Salud Publica/Pan Am/Public Health 3: 242-248.

16. Guía para la atención al paciente infectado con Trypanosoma cruzi. Ministerio de Salud, 2006. Resolución 1870. Available at: www.msal.gov.ar/htm/site/chagas. Accessed August 10, 2007.

17. Svahn A, Linde A, Thorstensson R, Karlen K, Andersson L, Gaines H, 2003. Development and evaluation of a flowcytometric assay of specific cell-mediated immune response in activated whole blood for the detection of cell-mediated immunity against varicella-zoster virus. J Immunol Methods 277: $17-25$.

18. Leeuwenberg JF, Jeunhomme TM, Buurman WA, 1994. Slow release of soluble TNF receptors by monocytes in vitro. J Immunol 152: 4036-4043.

19. Joyce DA, Gibbons DP, Green P, Steer JH, Feldmann M, Brennan FM, 1994. Two inhibitors of pro-inflammatory cytokine release, interleukin-10 and interleukin-4, have contrasting effects on release of soluble p75 tumor necrosis factor receptor by cultured monocytes. Eur J Immunol 24: 2699-2705.

20. Raghupathy R, 1997. Th1-type immunity is incompatible with successful pregnancy. Immunol Today 18: 478-482.

21. Steinborn A, Gunes H, Halberstadt E, 1995. Signal for term parturition is of trophoblast and therefore of fetal origin. Prostaglandins 50: 237-252.

22. Steinborn A, Gunes H, Roddiger S, Halberstadt E, 1996. Elevated placental cytokine release, a process associated with preterm labor in the absence of intrauterine infection. Obstet Gynecol 88: 534-539.

23. Joyce DA, Steer JH, Abraham LJ, 1997. Glucocorticoid modulation of human monocyte/macrophage function: control of TNF- $\alpha$ secretion. Inflamm Res 46: 447-451.

24. Snyder SK, Wessner DH, Wessells JL, Waterhouse RM, Wahl LM, Zimmermann W, Dveksler GS, 2001. Pregnancy-specific glycoproteins function as immunomodulators by inducing secretion of IL-10, IL- 6 and TGF- $\beta 1$ by human monocytes. Am J Reprod Immunol 45: 205-216.

25. Chernyshov VP, Vodyanik MA, Pisareva SP, 2005. Lack of soluble TNF-receptors in women with recurrent spontaneous abortion and possibility for its correction. Am J Reprod Immunol 54: 284-291.

26. Vekemans J, Truyens C, Torrico F, Solano M, Torrico MC, Rodriguez P, Alonso-Vega C, Carlier Y, 2000. Maternal Trypanosoma cruzi infection upregulates capacity of uninfected neonate cells to produce pro- and anti-inflammatory cytokines. Infect Immun 68: 5430-5434.

27. Kwiatkowski D, Hill AV, Sambou I, Twumasi P, Castracane J, Manogue KR, Cerami A, Brewster DR, Greenwood BM, 1990. TNF concentration in fatal cerebral, non-fatal cerebral, and uncomplicated Plasmodium falciparum malaria. Lancet 336: 1201-1204.

28. Zwingenberger K, Harms G, Pedrosa C, Pessoa MC, Sandkamp B, Scheibenbogen C, Andreesen R, 1991. Generation of cytokines in human visceral leishmaniasis: dissociation of endogenous TNF- $\alpha$ and IL-1 $\beta$ production. Immunobiology 183: 125132.

29. Shobokshi A, Shaarawy M, 2002. Maternal serum and amniotic fluid cytokines in patients with preterm premature rupture of membranes with and without intrauterine infection. Int $J G y$ naecol Obstet 79: 209-215.

30. Van Zee KJ, Kohno T, Fischer E, Rock CS, Moldawer LL, Lowry SF, 1992. Tumor necrosis factor soluble receptors circulate during experimental and clinical inflammation and can protect against excessive tumor necrosis factor $\alpha$ in vitro and in vivo. Proc Natl Acad Sci USA 89: 4845-4849.

31. Austgulen R, Liabakk NB, Brockhaus M, Espevik T, 1992. Soluble TNF receptors in amniotic fluid and in urine from pregnant women. J Reprod Immunol 22: 105-116.

32. Doellner H, Arntzen KJ, Haereid PE, Aag S, Brubakk AM, Austgulen R, 1998. Increased serum concentrations of soluble tumor necrosis factor receptors p55 and p75 in early onset neonatal sepsis. Early Hum Dev 52: 251-261. 